\title{
Ferric ion crosslinking-based 3D printing of graphene oxide and its evaluation as bio-scaffold in tissue engineering
}

\author{
Renhao Lu¹, Wuhua Zhang ${ }^{1}$, Yuting $\mathrm{He}^{2}$, Siyuan Zhang ${ }^{1}$, Qian Fu², Yuan Pang ${ }^{1}$, and Wei \\ Sun $^{1}$ \\ ${ }^{1}$ Tsinghua University \\ ${ }^{2}$ Chongqing University
}

May 6, 2020

\begin{abstract}
As a precursor of graphene, graphene oxide (GO) exhibits excellent mechanical, thermal, and electrical properties, besides appreciable biocompatibility in tissue engineering applications. However, the current GO-3D fabrication technology is still in need of optimization and simplification in order to ensure fine architecture and reasonable mechanical property, which would further promote the performance of GO as bio-scaffolds in cell or microorganism attachment and in material transformation. To address this issue, we proposed a GO ink, with appreciable rheological properties and excellent printing performance via high-speed centrifugation and ferric ion-assisted cross-linking. A woodpile structure with controllable micro-pores was produced by micro-extrusion-based 3D printing technology followed by an optimized freeze-drying process. Cellular adhesion and viability were verified by inoculation and culture of HepaRG cells using the fabricated GO 3D structure, thus suggesting ferric ion-assisted cross-linking and controllable pore distribution to improve the performance of GO construct as a bio-scaffold for in-vitro liver tissue models.
\end{abstract}

Ferric ion crosslinking-based 3D printing of graphene oxide and its evaluation as bio-scaffold in tissue engineering

Renhao $\mathrm{Lu}^{1, \#}$, Wuhua Zhang ${ }^{1}$, Yuting $\mathrm{He}^{2,3}$, Siyuan Zhang ${ }^{1}$, Qian Fu², ${ }^{2}$, Yuan Pang ${ }^{1,}$, , , Wei Sun ${ }^{1,4,5}$, *

${ }^{1}$ Biomanufacturing Center, Department of Mechanical Engineering, Tsinghua University, Haidian District, Beijing 100084, China

${ }^{2}$ Key Laboratory of Low-grade Energy Utilization Technologies and Systems, Chongqing University, Ministry of Education, Chongqing 400044, China

${ }^{3}$ Institute of Engineering Thermophysics, School of Energy and Power Engineering, Chongqing University, Chongqing 400044, China

${ }^{4}$ Biomanufacturing and Rapid Forming Technology Key Laboratory of Beijing, Beijing 100084, China

${ }^{5}$ Department of Mechanical Engineering, Drexel University, Philadelphia, PA, USA

\# Current affiliation: Nancy E. and Peter C. Meinig School of Biomedical Engineering, Cornell University, Ithaca, NY 14853, USA;

* Yuan Pang and Wei Sun contributed equally and are co-corresponding authors.

Corresponding authors:

Yuan Pang, Ph.D. 
Dept. of Mechanical Engineering, Tsinghua University, Room A730, Lee Shau Kee Science and Technology Building, Haidian District, Beijing 100084, P. R. China

Email: pangyuan31@mail.tsinghua.edu.cn, Tel: +86 15652179395

Wei Sun, Ph.D.

Dept. of Mechanical Engineering, Tsinghua University, Room A743, Lee Shau Kee Science and Technology Building, Haidian District, Beijing 100084, P. R. China.

Email: weisun@mail.tsinghua.edu.cn, sunwei@drexel.edu, Tel: +86 13522556809

Abstract As a precursor of graphene, graphene oxide (GO) exhibits excellent mechanical, thermal, and electrical properties, besides appreciable biocompatibility in tissue engineering applications. However, the current GO-3D fabrication technology is still in need of optimization and simplification in order to ensure fine architecture and reasonable mechanical property, which would further promote the performance of GO as bio-scaffolds in cell or microorganism attachment and in material transformation. To address this issue, we proposed a GO ink, with appreciable rheological properties and excellent printing performance via high-speed centrifugation and ferric ion-assisted cross-linking. A woodpile structure with controllable micro-pores was produced by micro-extrusion-based 3D printing technology followed by an optimized freezedrying process. Cellular adhesion and viability were verified by inoculation and culture of HepaRG cells using the fabricated GO 3D structure, thus suggesting ferric ion-assisted cross-linking and controllable pore distribution to improve the performance of GO construct as a bio-scaffold for in-vitro liver tissue models.

Keywords: graphene oxide, 3D printing, lyophilization, porous structure, liver tissue engineering

\section{Introduction}

As a single-layered carbon material, graphene exhibits high mechanical strength, large surface area, superior chemical stability, and extraordinary electrical and thermal conductivities, thus finding application in energy storage, sensors, etc. (Y. Zhu et al., 2010). Recently, graphene-based materials have shown promise as bio-scaffold owing to their biocompatibility, conductivity, anti-inflammatory properties, and differentiation effects during cell growth in multiple cell types (Sahni et al., 2013). The ultra-low density of graphene leads to easy biodegradation, and its high porosity enhances oxygen diffusion. All these factors collectively help graphene mimic the physiological environment better than many hydrogels and polymers (Loeblein et al., 2016). However, most of the potential applications require a large specific surface area for cells and chemical compounds to attach, along with a macrostructure to operate, connect, and examine. In order to circumvent the limitations of 2D materials and transfer the outstanding properties into three dimensions, there is an emerging need to assemble graphene into a 3D structure with specific dimensions and a well-controlled microstructure (C. Zhu et al., 2015). Since graphene is hydrophobic, it can be modified with oxygen-containing groups, such as hydroxyl and carboxyl groups, by a reversible oxidation process (Hummers' method), thereby forming graphene oxide (GO) (Hummers Jr \& Offeman, 1958), which has enhanced hydrophilicity, dispersity, and forming property. GO retains most properties of graphene, except conductivity, which can also be easily recovered by reduction reaction with hydroiodic acid or ascorbic acid. Transformation of GO into a 3D structure, based on aqueous solution or gel, can be easily controlled in an inexpensive way, thus making it a preferred method for assembling graphene-based materials toward multi-functional applications.

Conventional forming methods for graphene-based materials include hydrothermal method and chemical vapor deposition (CVD)(Xu, Sheng, Li, \& Shi, 2010), among many others. The hydrothermal method, developed by $\mathrm{Xu}$ et al, can fabricate self-assembled GO into 3D structure, with good conductivity and mechanical strength; however, the shape is defined by the container and pores are formed randomly (Xu et al., 2010). The graphene foam (GF), developed by Chen et al through CVD, is widely used as a bio-scaffold for hepatocytes, osteoblasts, chondrocytes, etc., and shows good biocompatibility and biodegradability (Loeblein et al., 2016; Xie et al., 2018; Yocham et al., 2018). Besides having similar disadvantages as the product from hydrothermal method, this material turns fragile under low pressure, and the method is not scalable. 
Compared to the above-mentioned methods, 3D printing possesses the ability to fabricate complicated and well-controlled 3D shapes through additive manufacture process with the help of computer-assisted design (CAD) (Wang, Jiang, Zhou, Gou, \& Hui, 2017). Mainly, two kinds of 3D printing technologies are used in GO printing: freeze-casting 3D printing and extrusion-based 3D-printing technique. Zhang et al had developed the freeze casting 3D printing method, which selectively solidifies the droplets of GO suspension into the cold sink $(-25)$ while the water and low-viscous GO suspension are printed in a drop-on-demand mode (Zhang et al., 2016). While the low concentration of GO and drop-by-drop printing can achieve high precision, it can also result in low mechanical strength of the structure, low speed of printing, and large deformation in lyophilization process. Extrusion-based 3D printing, on the other hand, directly accumulates the ink into the 3D structure with higher efficiency and strength. Nevertheless, this method requires high viscosity, high yield stress, and a shear-thinning behavior of the ink material (Jiang et al., 2018). Normally, GO aqueous solution is of low viscosity, and requires further treatments. Jakus et al and Yao et al had achieved printable GO ink property via concentration using evaporation and lyophilization, respectively; however, both the methods were highly time-consuming (Jakus et al., 2015; Yao et al., 2016). Centrifugation is a fast and inexpensive way for concentrating, although the highest achievable concentration depends on the speed limit of the centrifuge (Jiang et al., 2018; C. Zhu et al., 2015). Based on centrifugation, Zhu et al had used 10-20 wt\% silica as the viscosifier to increase the storage modulus of GO by an order of magnitude and achieve a highly compressible 3D structure (C. Zhu et al., 2015). However, silica is removed by hydrofluoric acid, which can, in turn, induce cytotoxicity. Jiang et al used $15 \mathrm{mmol} / \mathrm{L} \mathrm{CaCl}_{2}$ as a crosslinker and achieved highresolution printing (Jiang et al., 2018). Almost all mentioned 3D printing methods used lyophilization as the final treatment. However, the diameter, distribution, and orientation of pores, which are crucial parameters for cell attachment and proliferation, remain to be explored in detail. Multiple organic compounds (PEO, HPC, etc.), including divalent and trivalent ions $\left(\mathrm{Ca}^{2+}, \mathrm{Mg}^{2+}, \mathrm{Cu}^{2+}, \mathrm{Pb}^{2+}, \mathrm{Cr}^{3+}, \mathrm{Fe}^{3+}\right)$, have been proven to induce GO gelation; however, only seldom have been studied for rheological properties, printability, or toxicity (Bai, Li, Wang, \& Shi, 2011). Therefore, 3D printing of GO is still in need of further research and optimization, for designing a GO 3D structure with better microstructure and stable mechanical strength, for advanced application in tissue engineering. We herein proposed our work of improving the mechanical strength of GO by adding trivalent ions with optimized concentration as crosslinker. $\mathrm{Fe}^{3+}$ was selected due to its low cytotoxicity and high coordination number, and was used as crosslinker to fabricate the three-dimensional GO structure with both macro and micro porosity.

In order to characterize and optimize GO hydrogel, rheological properties of pure GO, following centrifugation, were tested first. As shown in fig. 1a, oscillatory measurements proved GO to be in gel state under low shear stress, with a storage modulus of $519 \mathrm{~Pa}$ and loss modulus of $111 \mathrm{~Pa}$. The sol-gel transformation point was $50.5 \mathrm{~Pa}$. Rotational measure proved the shear thinning behavior of GO hydrogel (fig. 1b), and the shear stress-shear rate relation coincided with the Herschel-Bulkley non-Newtonian fluid model (fig. 1c) (Truby \& Lewis, 2016). A yield stress of $56.9 \mathrm{~Pa}$ was calculated by curve fitting.

\begin{tabular}{ll}
\hline$\tau=\tau_{\mathrm{HB}}+\eta_{\mathrm{HB}} \dot{\gamma}^{p}$ & $(1)$ \\
\hline$\tau_{\mathrm{HB}}=56.86 \mathrm{~Pa}, \eta_{\mathrm{HB}}=2.015, p=0.7528, R^{2}=1$ & $(2)$ \\
\hline
\end{tabular}

Where $\tau$ is the shear stress, $\tau_{\mathrm{HB}}$ is the shear yield stress, $\eta_{\mathrm{HB}}$ is the consistency, $\gamma$ is the shear rate and $p$ is the flow index ( $<1$ for shear thinning fluids). Theoretically, Herschel-Bulkley fluid has the ability to hold the shape owing to the shear yield stress, which is suitable for extrusion-based 3D printing method. However, 3D printing test with pure GO hydrogel failed with severe collapse owing to the low mechanical strength (data not shown).

Considering the ion crosslinking theory mentioned previously, we chose ferric ion as the crosslinker, owing to its low toxicity and high charge number. In order to find the optimal concentration, we tested the rheology and printability of GO with $\mathrm{Fe}^{3+}$ over the concentration range of $0-8 \mathrm{mmol} / \mathrm{L}$, since the solubility of $\mathrm{Fe}^{3+}$ is $8 \mathrm{mmol} / \mathrm{L}$. According to the result in fig. 1d, the GO hydrogel possessed higher elastic modulus and high 
shear yield stress at increased concentrations. Compared to that of pure GO hydrogel, both storage modulus and loss modulus of $\mathrm{Fe}^{3+}$-modified hydrogel increased by 1.7 orders of magnitude (fig. 1e). Printability was evaluated according to equation (3), developed by Ouyang et al. (Ouyang, Yao, Zhao, \& Sun, 2016).

$\overline{\operatorname{Pr}=\frac{\pi}{4} \bullet \frac{1}{C}=\frac{L^{2}}{16 A} \quad(3)}$

Where C, L, and A are the circularity, perimeter, and area of the grids respectively. For 3D printing of hydrogel, the ideal gelation condition should demonstrate square shape of grids $(\operatorname{Pr}=1)$, with higher and lower $\operatorname{Pr}$ values representing over-gelation and under-gelation states, respectively. In fig. $1 \mathrm{f} \& 1 \mathrm{~g}$, the measured printability coincided with rheology results, in which $8 \mathrm{mmol} / \mathrm{L}$ samples showed $\mathrm{Pr}$ values closest to 1 (proper gelation). $\mathrm{Fe}^{3+}$ ion, at the concentration of $8 \mathrm{mmol} / \mathrm{L}$, exhibited the best results in both rheology and printability, which was, therefore, selected as the optimal concentration for further printing.

Then, structures with different filament distances ( $1 \mathrm{~mm}, 1.5 \mathrm{~mm}, 2 \mathrm{~mm}, 2.5 \mathrm{~mm}$, and $3 \mathrm{~mm})$ were printed in order to test the forming property of $\mathrm{Fe}^{3+}$-crosslinked $\mathrm{GO}$ hydrogel under different printing conditions. During the printing process, parameters including the nozzle diameter, extrusion speed, scanning speed, and nozzle height were adjusted to achieve an optimized printed structure. The grid within the printed network became a macroporous structure. Ultimately, under the conditions of all filament distances, an intact structure with three-way pores (along x, y, and z axes in fig. 2b) was obtained (fig. 2c).

Based on the stable and well-controlled structure with the help of $\mathrm{Fe}^{3+}$ ion, lyophilization properties could be further studied. There are three steps in lyophilization: freezing, main drying, and final drying. Printed structures were frozen under -80 and -20 to explore the relationship between freezing temperature and pore size. Pore structure with a mean diameter of $11.19 \pm 5.46 \mu \mathrm{m}(\mathrm{n}=107)$ was produced by freeze-drying at a uniform temperature field of -80 (fig. 3a, 3b). For -20 field, the diameter of pores was $22.41 \pm 11.55$ $\mu \mathrm{m}(\mathrm{n}=93)$ (fig. 3d, 3e). The distribution of pore size, as shown in fig. 3c \& 3f, can be roughly fitted into Gaussian distribution. Additionally, samples were frozen at the temperature gradient field to test their feasibility to form oriented porous structures (fig. 3g, 3h, and 3i). Oriented pores with a mean width of $86.7 \pm 19.0 \mu \mathrm{m}$ were observed therein. During the freeze drying process, ice crystals were formed in the freezing stage that sublimed in the main drying stage. As a result, the space that used to be occupied by ice crystals became pores after lyophilization, shape, and size of the pores being decided by the crystallization process, and ultimately controlled by the freezing parameters. Compared to those obtained by freezing at -80 , we obtained pores of approximately double the diameter $(22.41 \mu \mathrm{m}$ vs. $11.19 \mu \mathrm{m})$ at a higher freezing temperature $(-20)$, which is in line with the mechanism of crystalline growth. The oriented pores in fig. 3g, $3 \mathrm{~h}$, and $3 \mathrm{i}$ proved the temperature gradient field to successfully guide the growth direction of ice crystal.

Cell attachment experiment was then conducted to test the performance of GO structures as a bio-scaffold using Human hepatoma cell line HepaRG, which is able to be differentiated and applied to build liver tissue model. According to the live/dead assay (fig. 4), we found HepaRG cells to have good viability (of $91.0 \pm$ $7.2 \%$ ) in the GO scaffold, as evident from the assistance of enhanced oxygen and nutrient transportation through the porous structures. More attached cells were found near the grid area, both on the surface and interior, which proved that the macro pores increased the specific surface area for cell attachment. According to DNA content measurement, the immobilized cells were approximately $3.06 \times 10^{6}$ cells $/ \mathrm{cm}^{3}$ in the GO scaffold. However, during z-axis scanning, few cells were detected in the interior, which could be attributed to either the opaqueness of graphene oxide or less cell distribution within. Therefore, the current thickness of $4 \mathrm{~mm}$ of the GO scaffold might be too far for the cell to travel through, even with the help of macro and micro pores; further improvement in design is expected in the future.

Appreciable cell viability and attachment behavior proved the biocompatibility of $\mathrm{Fe}^{3+}$-crosslinked GO structures and showed that $\mathrm{Fe}^{3+}$ at the concentration [?] $8 \mathrm{mmol} / \mathrm{L}$ does not induce cytotoxicity. Consequently, such GO structures can be applied as promising bio-scaffold in tissue engineering. The remaining problem was the immobilized cell density, which was not comparable in magnitude to that seen in vivo (10 $0^{8}$ to $10^{9}$ 
cells $/ \mathrm{cm}^{3}$ ) due to the thick structure along the z-axis. Hence, further geometric optimization would be helpful for higher cell density. Two-dimensional (2D) graphene materials had demonstrated the capability of pre-concentrating the differentiation inducers, thus accelerating the differentiation of human mesenchymal stem cells (MSCs) (Lee et al., 2011). Therefore, we expect the differentiation of HepaRG into hepatocytes and bile duct cells could also be enhanced with the effect of concentrating dimethylsulfoxide (DMSO) in the 3D GO structure (Guillouzo et al., 2007). Moreover, 3D graphene showed anti-inflammatory ability in microglial cells while 2D graphene failed to do so (Song et al., 2014). Our GO scaffold may also be used to culture neural stem cells owing to the same property. All these novel properties are beneficial to future applications of GO scaffold in multiple cell types.

In summary, we improved the rheological behavior and printability of graphene oxide hydrogel by inducing ferric ion as the crosslinker and using micro extrusion-based 3D printing technology to 3D-print the hydrogel, at room temperature, with a wide range of filament distances and diameters. Additionally, controllable porous structure inside the graphene structures was achieved by varying the freezing parameters in lyophilization process. The $\mathrm{Fe}^{3+}$-crosslinked GO structure is of low cytotoxicity, on which HepaRG cells showed good viability and attachment behavior. Considering the excellent properties of graphene materials in cell culture and their anti-inflammatory effects, the current stable, well-controlled, porous, and easily fabricated GO structure would be a promising bio-scaffold for developingin-vitro liver tissue models.

\section{Materials and methods}

Preparation and characterization of GO hydrogel The GO suspension was purchased from Suzhou Tanfeng Graphene Technology Co., Ltd,Suzhou, China, centration of $10 \mathrm{mg} / \mathrm{mL}$ and graphene diameter between 0.5 and $5 \mu \mathrm{m}$. $\mathrm{FeCl}_{3} \cdot 6 \mathrm{H}_{2} \mathrm{O}$ (Macklin) was dissolved in deionized water to prepare a $0.5 \mathrm{mmol} / \mathrm{L}$ stock. GO suspension was vialed into $50-\mathrm{mL}$ centrifuge tubes and concentrated up to $31 \mathrm{mg} / \mathrm{mL}$ by centrifuging at 9500 $\mathrm{rpm}$ for $30 \mathrm{~min}$. After removal of the supernatant, the hydrogel was mixed with $\mathrm{FeCl}_{3}$ and stirred for $20 \mathrm{~min}$. $\mathrm{FeCl}_{3}$ particles would appear at the beginning and dissolve gradually with stirring when the concentration of $\mathrm{Fe}^{3+}$ was [?] $8 \mathrm{mmol} / \mathrm{L}$ in GO hydrogel. Rheology of the prepared GO hydrogel was tested by the rheometer MCR 302 (Anton Paar GmbH).

$3 D$ printing set up Optimized GO hydrogel was collected into 1-mL syringes with nozzles (inner diameter ranging from $0.11 \mu \mathrm{m}$ to $0.42 \mu \mathrm{m}$ ) (fig. 2a). After assembling the GO-loaded syringe onto the desktop cell 3D printer ALPHA-CPD1 (SunP Biotech Co. Ltd.), the GO hydrogel was printed into woodpile structure, with a $20 \mathrm{~mm} \times 20 \mathrm{~mm} \times 4 \mathrm{~mm}$ cuboid dimension, at room temperature. The printability of the hydrogel was verified by printing with different filament distances, from $1 \mathrm{~mm}$ to $3 \mathrm{~mm}$.

Lyophilizing process and porous structure control The well-controlled unidirectional temperature field from -20 to room temperature was established within a custom-made mold as previously described (Fang, Zhang, Zhang, Gong, \& Sun, 2019). After freezing, all samples were then dried at $-50 \mathrm{degC}$ and 0.05 mbar for 48 $\mathrm{h}$ in a freeze drying machine Alpha 1-2 LDplus (Martin Christ Gefriertrocknungsanlagen GmbH, Osterode am Harz, Germany). Afterward, Samples were observed under scanning electron microscope (SEM), and diameters were measured by Image Pro Plus 6.0 software.

Cell adhesion measurement The culture medium was composed of Dulbecco's modified Eagle's Medium (DMEM) and supplemented with $10 \%$ fetal bovine serum (FBS), $0.05 \%$ insulin, $5 \times 10^{-5} \mathrm{~mol} / \mathrm{L}$ hydrocortisone hemisuccinate, $1 \%$ penicillin-streptomycin, $1 \%$ Gluta Max supplement, and 1\% MEM nonessential amino acid solution (NEAA). Printed samples with filament distance of $1 \mathrm{~mm}$ and uniform freezing condition of -80 , were used for the cell experiments. For sterilization, GO samples were soaked in $70 \%$ ethanol for $15 \mathrm{~min}$ under UV light and then transferred to PBS on a rotary shaker for 45 min to elute the residual ethanol. For cell attachment experiments, collagen type I was coated on the GO structure to improve the biocompatibility and cell attachment. Briefly, the sterilized sample was incubated in $0.3 \mathrm{mg} / \mathrm{mL}$ collagen solution for $1 \mathrm{~h}$ and washed with PBS. The samples were moved to untreated 6 -well plates with $2 \mathrm{~mL}$ of $1 \times 10^{6} / \mathrm{mL}$ HepaRG cell suspension in each well. After two days of co-culture, cell attachment and viability were evaluated by live/dead assay. The samples were carefully washed with PBS and then stained with calcein acetoxymethyl 
(calcein AM) and propidium iodide (PI) to identify the live-cell condition. The samples were subsequently observed under a confocal microscope.

Conflicts of Interest: The authors have no conflicts of interest to declare.

\section{Acknowledgements}

This work was supported by the National Natural Science Foundation of China (51805294), Tsinghua University Initiative Scientific Research Program (20197050024), Tsinghua University-Peking Union Medical College Hospital Initiative Scientific Research Program (20191080843), the National Key Research and Development Program of China (2018YFA0703004), and the 111 Project (G2017002).

\section{References}

Bai, H., Li, C., Wang, X., \& Shi, G. (2011). On the gelation of graphene oxide. The Journal of Physical Chemistry C, 115 (13), 5545-5551.

Fang, Y., Zhang, T., Zhang, L., Gong, W., \& Sun, W. (2019). Biomimetic design and fabrication of scaffolds integrating oriented micro-pores with branched channel networks for myocardial tissue engineering.Biofabrication, 11 (3), 035004. doi:10.1088/1758-5090/ab0fd3

Guillouzo, A., Corlu, A., Aninat, C., Glaise, D., Morel, F., \& Guguen-Guillouzo, C. (2007). The human hepatoma HepaRG cells: a highly differentiated model for studies of liver metabolism and toxicity of xenobiotics. Chem Biol Interact, 168 (1), 66-73. doi:10.1016/j.cbi.2006.12.003

Hummers Jr, W. S., \& Offeman, R. E. (1958). Preparation of graphitic oxide. Journal of the american chemical society, 80 (6), 1339-1339.

Jakus, A. E., Secor, E. B., Rutz, A. L., Jordan, S. W., Hersam, M. C., \& Shah, R. N. (2015). Threedimensional printing of high-content graphene scaffolds for electronic and biomedical applications. ACS nano, 9 (4), 4636-4648. doi:10.1021/acsnano.5b01179

Jiang, Y., Xu, Z., Huang, T., Liu, Y., Guo, F., Xi, J., . . . Gao, C. (2018). Direct 3D printing of ultralight graphene oxide aerogel microlattices. Advanced Functional Materials, 28 (16), 1707024.

Lee, W. C., Lim, C. H., Shi, H., Tang, L. A., Wang, Y., Lim, C. T., \& Loh, K. P. (2011). Origin of enhanced stem cell growth and differentiation on graphene and graphene oxide. ACS nano, 5 (9), 73347341. doi: $10.1021 / \mathrm{nn} 202190 \mathrm{c}$

Loeblein, M., Perry, G., Tsang, S. H., Xiao, W., Collard, D., Coquet, P., . . . Teo, E. H. (2016). ThreeDimensional Graphene: A Biocompatible and Biodegradable Scaffold with Enhanced Oxygenation. Adv Healthc Mater, 5 (10), 1177-1191. doi:10.1002/adhm.201501026

Ouyang, L., Yao, R., Zhao, Y., \& Sun, W. (2016). Effect of bioink properties on printability and cell viability for 3D bioplotting of embryonic stem cells. Biofabrication, 8 (3), 035020. doi:10.1088/1758-5090/8/3/035020

Sahni, D., Jea, A., Mata, J. A., Marcano, D. C., Sivaganesan, A., Berlin, J. M., . . . Tour, J. M. (2013). Biocompatibility of pristine graphene for neuronal interface. J Neurosurg Pediatr, 11 (5), 575-583. doi:10.3171/2013.1.PEDS12374

Song, Q., Jiang, Z., Li, N., Liu, P., Liu, L., Tang, M., \& Cheng, G. (2014). Anti-inflammatory effects of three-dimensional graphene foams cultured with microglial cells. Biomaterials, 35 (25), 6930-6940. doi:10.1016/j.biomaterials.2014.05.002

Truby, R. L., \& Lewis, J. A. (2016). Printing soft matter in three dimensions. Nature, 540 (7633), 371-378. doi:10.1038/nature21003

Wang, X., Jiang, M., Zhou, Z., Gou, J., \& Hui, D. (2017). 3D printing of polymer matrix composites: A review and prospective. Composites Part B: Engineering, 110 , 442-458. 
Xie, W., Song, F., Wang, R., Sun, S., Li, M., Fan, Z., . . . Wang, J. (2018). Mechanically robust 3D graphene-hydroxyapatite hybrid bioscaffolds with enhanced osteoconductive and biocompatible performance. Crystals, 8 (2), 105.

Xu, Y., Sheng, K., Li, C., \& Shi, G. (2010). Self-assembled graphene hydrogel via a one-step hydrothermal process. ACS nano, 4 (7), 4324-4330. doi:10.1021/nn101187z

Yao, Y., Fu, K. K., Yan, C., Dai, J., Chen, Y., Wang, Y., . . . Hu, L. (2016). Three-Dimensional Printable High-Temperature and High-Rate Heaters. ACS nano, 10 (5), 5272-5279. doi:10.1021/acsnano.6b01059

Yocham, K. M., Scott, C., Fujimoto, K., Brown, R., Tanasse, E., Oxford, J. T., . . . Estrada, D. (2018). Mechanical Properties of Graphene Foam and Graphene Foam-Tissue Composites. Advanced engineering materials, 20 (9), 1800166

Zhang, Q., Zhang, F., Medarametla, S. P., Li, H., Zhou, C., \& Lin, D. (2016). 3D Printing of Graphene Aerogels. Small, 12 (13), 1702-1708. doi:10.1002/smll.201503524

Zhu, C., Han, T. Y., Duoss, E. B., Golobic, A. M., Kuntz, J. D., Spadaccini, C. M., \& Worsley, M. A. (2015). Highly compressible 3D periodic graphene aerogel microlattices. Nat Commun, 6 (1), 6962. doi:10.1038/ncomms7962

Zhu, Y., Murali, S., Cai, W., Li, X., Suk, J. W., Potts, J. R., \& Ruoff, R. S. (2010). Graphene and graphene oxide: synthesis, properties, and applications. Adv Mater, 22 (35), 3906-3924. doi:10.1002/adma.201001068

\section{Figure legends}

Fig. 1 (a-c) Rheological properties of pure GO hydrogel: (a) Complex modulus as a function of shear stress; (b) Shear stress and (c) dynamic viscosity as functions of shear rate. (d) Elastic modulus and yield stress of GO hydrogel under different $\mathrm{Fe}^{3+}$ concentrations. (e) Complex modulus of pure $\mathrm{GO}$ and $\mathrm{Fe}^{3+}$-modified GO hydrogel. (f) Printability and (e) grid shape of GO hydrogel at different $\mathrm{Fe}^{3+}$ concentrations under an optical microscope. Scale bar, $500 \mu \mathrm{m}$.

Fig. 2 3D printing from hydrogel to 3D contruct. (a) GO hydrogel before and after $\mathrm{Fe}^{3+}$ modification. (b) Schematic illustration of three-way porous structure. (c) Intact contructs with different filament distances. Scale bar, $2 \mathrm{~mm}$.

Fig. 3 Porous structures under different freeze drying conditions. Uniform $-80{ }^{\circ} \mathrm{C}$ field: $(\mathrm{a}, \mathrm{b}) \mathrm{SEM}$ images and (c) distribution of pore sizes. Uniform $-20^{\circ} \mathrm{C}$ field: (d, e) SEM images and (f) distribution of pore sizes. Oriented temperature field: (g-i) SEM images. Scale bar, (a, d) 1 mm; (b, e) $100 \mu \mathrm{m}$; (g, h) $500 \mu \mathrm{m}$; (i) 200 $\mu \mathrm{m}$.

Fig. 4 Live/dead assay of HepaRG cell attachment on GO contructs. (a) Live cells, (b) dead cells, and (c) merged image. Scale bar, $200 \mu \mathrm{m}$. 

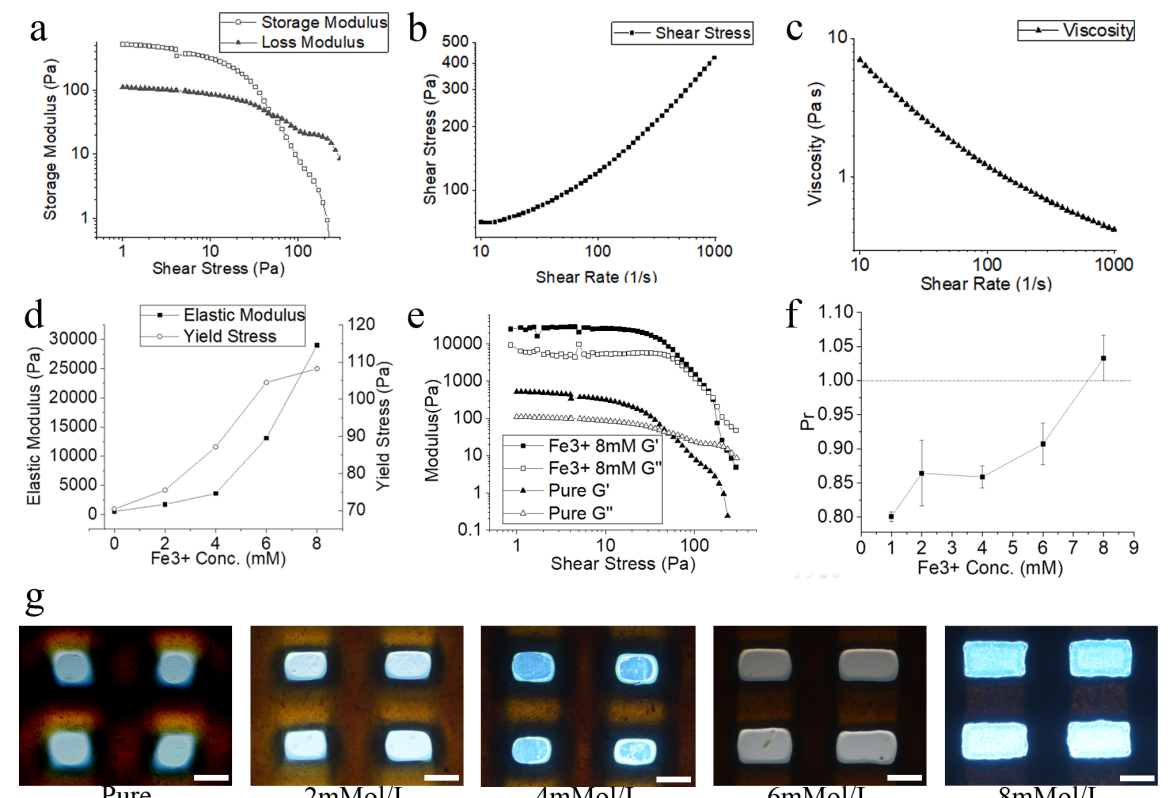

$6 \mathrm{mMol} / \mathrm{L}$

$8 \mathrm{mMol} / \mathrm{L}$
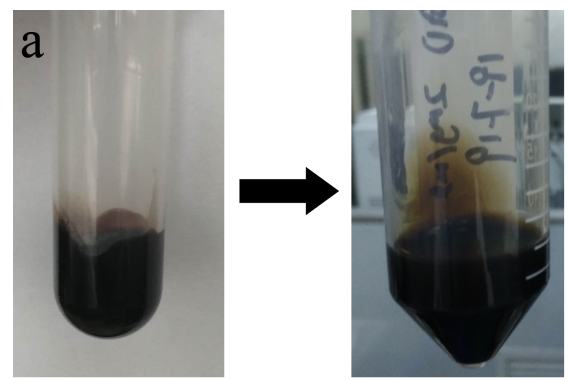

b
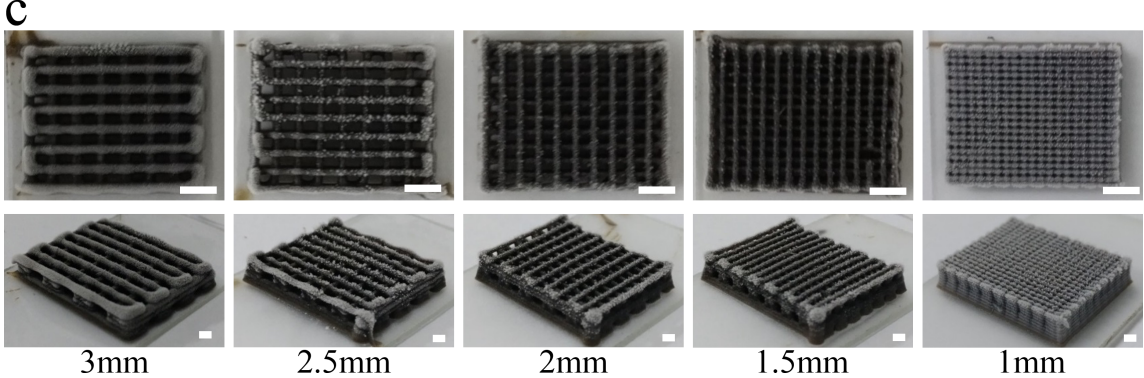

$1.5 \mathrm{~mm}$

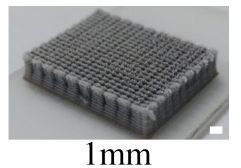



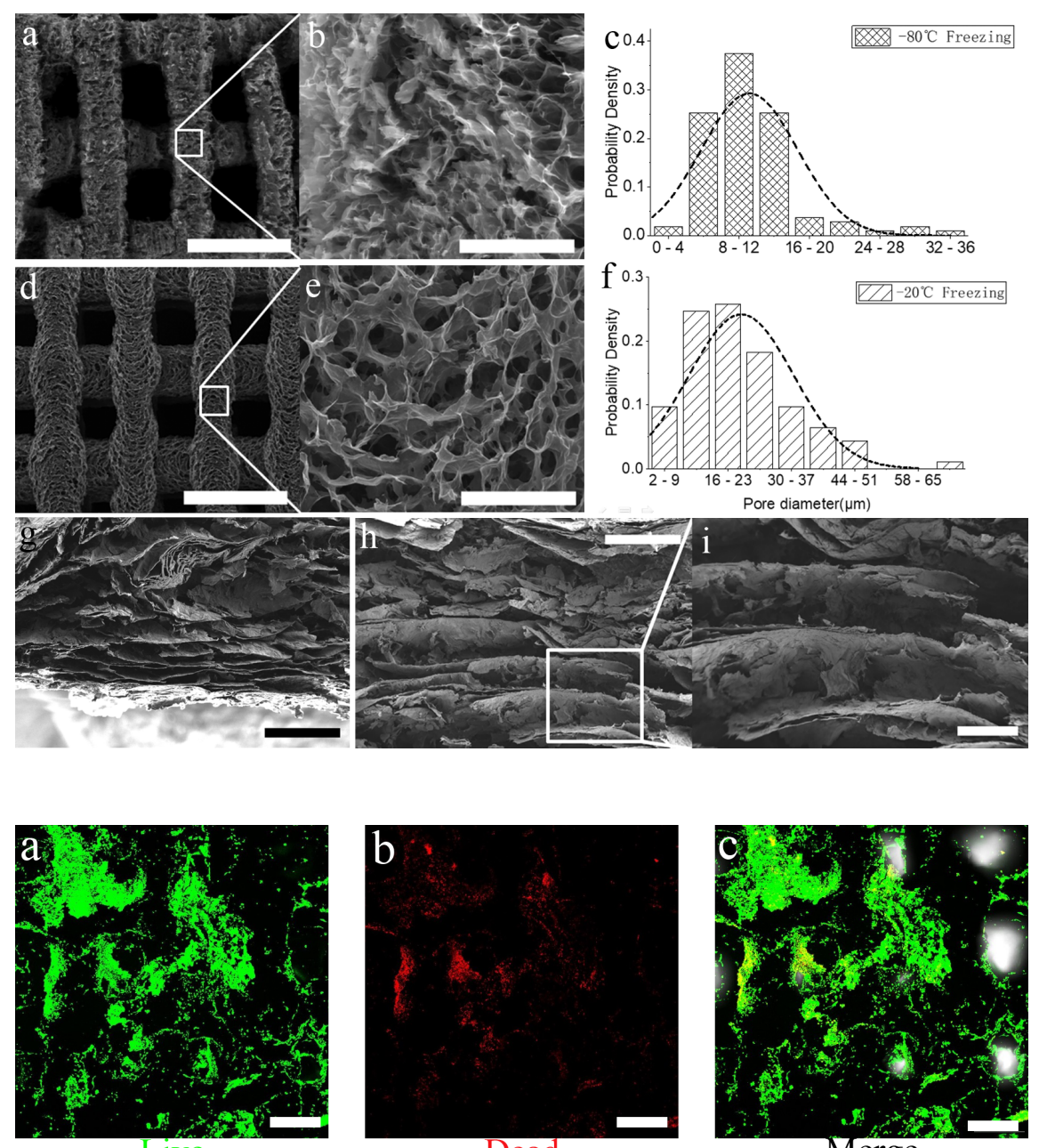

Dead

Merge 